\title{
HIGH ASPECT RATIO PARYLENE C MICROPILLARS FORMED BY MOLDING AND ION- BEAM ETCHING METHOD
}

\author{
Zdenka FOHLEROVA ${ }^{1,2^{*}}$, Peter FECKO ${ }^{2}$, Imrich GABLECH ${ }^{1,2}$, Zuzana KOSELOVA ${ }^{2}$ \\ ${ }^{1}$ Central European Institute of Technology, Brno, Czech Republic, EU, zdenka.fohlerova@ceitec.vutbr.cz \\ ${ }^{2}$ University of Technology, Faculty of Electrical Engineering and Communication, Brno, Czech Republic, EU
}

https://doi.org/10.37904/nanocon.2021.4349

\begin{abstract}
This paper represents the molding and plasma etching method for developing a high aspect ratio parylene C pillar array. The silicon mold was fabricated using the modified Bosch process. The parylene was deposited by chemical vapor deposition into the silicone holes, followed by the etching of silicon to obtain the transparent membrane of hollow parylene $C$ pillars. The etching method was initialized by transferring the pattern on the hard titanium mask via standard photolithography, followed by titanium etching. The oxygen plasma ion-milling of parylene $C$ produced the flexible pillars standing on the silicon substrate. Both pillar arrays were characterized by scanning electron microscopy. Arrays of micropillars could be applicable for measuring cellular forces or as the bioinspired platform with modulated surface chemistry.
\end{abstract}

Keywords: Parylene C, micropillars, molding, etching

\section{INTRODUCTION}

A high aspect ratio polymeric micro- and nanopillar arrays have attracted great attention in the fields of optics due to the ability to influence the propagation of light, in surface chemistry by modifying the bulk substrate wettability, controlling the cell attachment or estimation of cellular force in the field of biology [1]. The polymeric pillars have been prepared from various materials, including PDMS, photocurable, or thermoplastic polymers [2]. The properties of the individual polymer and the final high aspect ratio of pillars predetermine the fabrication technique and route of pillar array development. The common techniques for the fabrication of micro- and nanopillars are lithography techniques (UV, X-ray, or electron/ion-beam), molding and imprinting methods, plasma etching or laser ablation [3,4]. They differ in many aspects such as resolution, high aspect ratio, directionality, cost-effectiveness, low/high throughput or processed polymeric material, etc. For example, the molding technique of high aspect ratio polymeric pillars is challenging as the demolding process can damage pillars. Moreover, a high pillar density may lead to the structure collapse. Imprinting techniques require UV or thermally processable polymers, the etching process is limited to the availability of selective polymer etchant, lithographic techniques are expensive, etc.

Recently, parylene $\mathrm{C}$ got attention in biomedicine as the biocompatible polymer with high Young's modulus ( $\approx 4 \mathrm{GPa}$ ) [5]. Compared to the other polymers, parylene $\mathrm{C}$ must be deposited by a technique such as chemical vapor deposition, which has the disadvantage of higher requirement on the instrumentation. The fabrication of a high aspect ratio $\mathrm{SiO}_{2}$ decorated micropillar array by parylene $\mathrm{C}$ plasma etching has been introduced previously by our group [6]. Such pillar development requires a high degree of etching control and anisotropy as they are more prone to loss of features due to undercutting. Moreover, the durability of the etch mask in the plasma process is a key factor for achieving high aspect ratios. Thus, the metallic hard mask is utilized in most cases.

On the other hand, molding methods have been frequently used to develop lower high aspect ratio PDMS pillars. The development of parylene $\mathrm{C}$ pillars by molding technique appears challenging, but we previously showed the fabrication of bioinspired high aspect ratio $\mathrm{SiO}_{2}$ coated parylene pillars by molding technique [7]. 
More complex etch processes have to be utilized to prepare silicon mold to increase the aspect ratio. For such a purpose, the Bosch process introduces the solution of many repeated cycles between an isotropic $\mathrm{SF}_{6}$ etch and the deposition of a passivation polymer layer from $\mathrm{C}_{4} \mathrm{~F}_{8}$ gas to provide anisotropy.

This paper introduces the high aspect ratio parylene $C$ micropillars developed by the ion-beam etching method and replica molding technique combined with the modified Bosch process. Micropillars geometry was characterized by scanning electron microscopy (SEM), and both techniques were briefly discussed.

\section{METHODS}

\subsection{Dry etching method}

$A \approx 10 \mu \mathrm{m}$ thick layer of parylene $C$ was deposited on the $\mathrm{Si}(100)$ wafer using the chemical vapor deposition (CVD) method. A $300 \mathrm{~nm}$ thin titanium layer was deposited using the electron beam evaporation technique. UV photolithography was performed using the $\approx 1.4 \mu \mathrm{m}$ thick layer of photoresist (PR) AZ 5214E via mask aligner in vacuum contact mode (Figures 1-1A). The pattern of hexagonally arranged features with a diameter of $2 \mu \mathrm{m}$ and center-to-center distance of $4 \mu \mathrm{m}$ was developed. Then, the titanium was etched using reactive ion etching (RIE) in $\mathrm{Cl}_{2}$ plasma (Figures 1-1B). Finally, the wafer was placed into the ion beam etching (IBE) instrument employing Kaufman ion-beam source with collimated grids using pure $\mathrm{O}_{2}$ plasma for etching the parylene $\mathrm{C}$ from the areas uncovered by $\mathrm{Ti}$. The PR was completely removed during the IBE process, and the Ti residues were additionally removed using chlorine-based RIE (Figures 1-1C). The wafer was cut into the samples with dimensions of $(2 \times 2) \mathrm{cm}^{2}$ using a femtosecond laser to eliminate the generation of impurities.

\subsection{Molding method}

The parylene micropillars have been fabricated by molding method using Si mold (Figures 1-2A). The pattern was transferred to the $\mathrm{Si}$ wafer with $\approx 300 \mathrm{~nm}$ thermal $\mathrm{SiO}_{2}$ film and $\approx 1.5 \mu \mathrm{m}$ positive photoresist (PR) using contact lithography. The PR was baked at $120^{\circ} \mathrm{C}$ for $1 \mathrm{~min}$ and developed in a tetramethylammonium hydroxide solution. The $\mathrm{SiO}_{2}$ was then etched by the deep reactive ion etching (DRIE) method in inductively coupled plasma $\left(\mathrm{C}_{4} \mathrm{~F}_{8} / \mathrm{O}_{2}\right)$ for 1 minute (Figures 1-2B). The circular holes with a diameter and pitch of $1.6 \mu \mathrm{m}$ and $4 \mu \mathrm{m}$, respectively, were created by etching of $\approx 0.535 \mathrm{~mm}$ thick $\mathrm{Si}(100)$ substrate with the Bosch process (Figures 1-2C). This process provided a rigid mold of hexagonally arranged holes of a depth of $\approx 11 \mu \mathrm{m}$ (Figures 1-2D). PR residues were then removed by $\mathrm{O}_{2}$ plasma, and silicon oxide was removed with $49 \% \mathrm{HF}$ solution. The Si platform served as the mold for parylene $\mathrm{C}$ deposition under low pressure $\approx 1.6 \mathrm{~Pa}$ to improve coating uniformity (Figures 1-2E). The Si mold was etched in $\mathrm{XeF}_{2}$ vapors for $10 \mathrm{~s}$ to obtain the silicon chamber with exposed micropillar membrane (Figures 1- 2F).

1. Etching method

A)

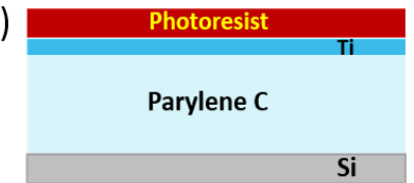

B)

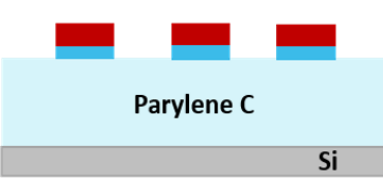

C)

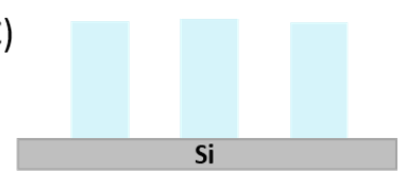

2. Molding method

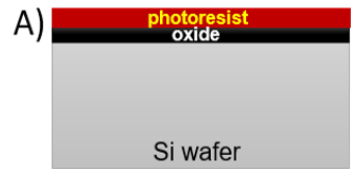

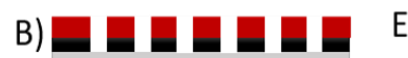
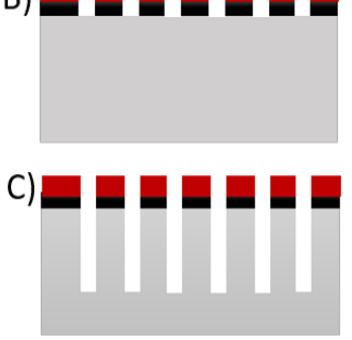

D)
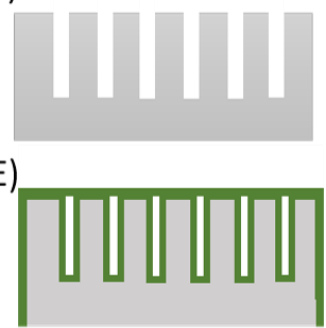

F)

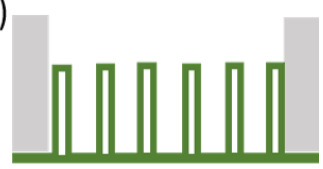

Figure 1 Scheme of fabrication of parylene $C$ micropillars via molding (1 AC) and etching (2A-F) techniques 


\section{RESULTS AND DISCUSSION}

\subsection{Etching technique}

Utilization of chemical dissolvent is possible for etching organic polymers in anisotropic way, but the parylene $\mathrm{C}$, which is chemically inert in practice, the only option that remains is plasma-based dry etching. Preliminary experiments on ion-beam etching systems (IBE) using $\mathrm{O} 2$ plasma were carried out. The parylene $\mathrm{C}$ deposited on $\mathrm{Si}$ substrate was covered with evaporated titanium film and micropatterned using standard photolithography. The hard titanium mask was subsequently etched with chlorine-based plasma followed by oxygen plasma etching of parylene (Figure 2A) [6]. In Figure 2B, the nano-forest formation can be visible on the bottom of the substrate, which is supposed to arise from the micro-/nano-masking effect of the sputtered titanium metal mask. To overcome this problem, introducing $\mathrm{SF}_{6}$ gas into the plasma source can counteract this masking phenomenon. However, the SF6 ion-beam suffered from poor directionality even at maximum allowed accelerator voltages, which yielded poor results [8]. However, inorganic hard masks lead to significant problems such as debris, sidewall roughening and grass formation, interaction with a polymer layer, scumming, and pattern distortion due to stress. Next, the slightly conical shape of pillars has been observed (Figure 2A) due to the ion-beam directionality of IBE. Nevertheless, the ideal cylindrical shape is beyond the equipment's capabilities we used. The top-down process of micropillar development provided the array of the hexagonally ordered parylene pillars with $\mathrm{a} \approx 1.6 \mu \mathrm{m}$ diameter, $\approx 4 \mu \mathrm{m}$ center-to-center distance, $\approx 9 \mu \mathrm{m}$ length, and rod-like morphology, as confirmed by SEM.

\subsection{Molding method}

The hexagonally arranged hollow parylene $C$ micropillars have been developed by molding method as shown in Figures $\mathbf{2 C}$, D. The pillar geometry was obtained of the diameter of $\approx 2 \mu \mathrm{m}$, pitch $\approx 4 \mu \mathrm{m}$, and the length of $\approx 11 \mu \mathrm{m}$. Using the Bosch process, a significant problem when applying this process can be the characteristic scalloping on the sidewall due to the isotropic etch step [9]. From SEM images is evident that we managed to minimalize this effect to a minimum. However, a slight scallop is visible (Figure 2D). Further, during the DRIE process of $\mathrm{SiO}_{2}$ and $\mathrm{Si}$, controlling the taper angle plays an important role. We optimized the angle during the Bosch process, and we got etched holes with a positive taper angle of $\approx 89^{\circ}$ instead of $\approx 90^{\circ}$, which resulted in the narrowing of holes to $\approx 1,6 \mu \mathrm{m}$. Tapering in the HAR Bosch process can be controlled by balancing the passivation step and the breakthrough step, i.e., decreasing passivation time or increasing breakthrough time to reduce positive tapering and vice versa. Thus, further optimization of the Bosch process is necessary to obtain a perfectly cylindrical hole of a high depth-to-diameter ratio. The optimization process with the development of high aspect ratio pillars complicates the effectiveness of high-energy ions. During the breakthrough step, ion energy decreases with depth due to particle collisions in plasma and an opposite electrostatic charge of vertical walls. Therefore, the ion energy can be more precisely controlled by advanced modulating of the plasma parameters of the Bosch process.

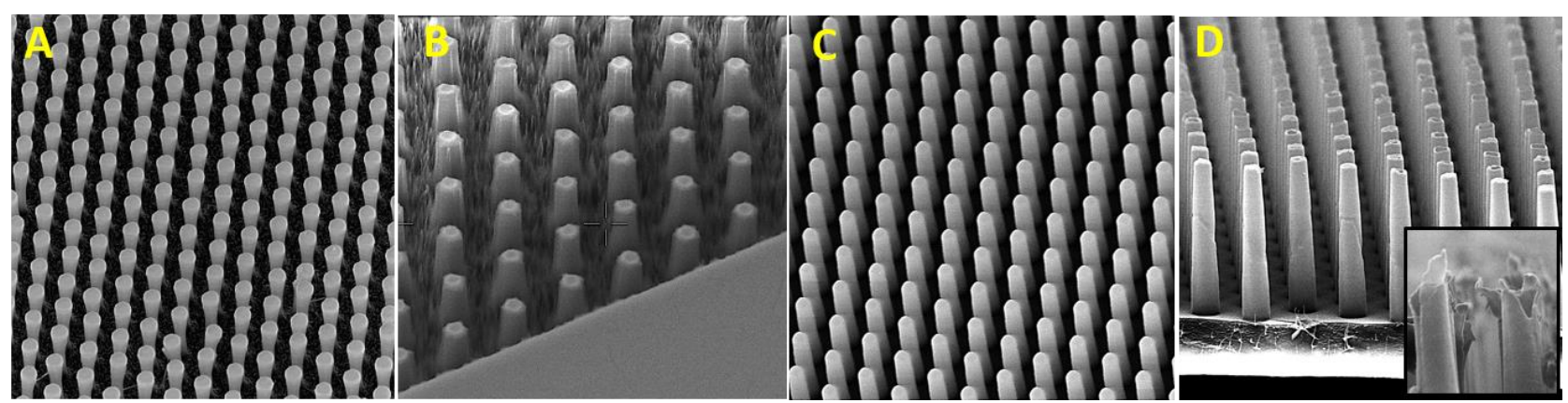

Figure 2 SEM image of parylene $C$ micropillars fabricated by etching method (A). Nanoforest from hard mask (B). SEM image of micropillars fabricated by molding method (C). Scallop effect on pillar surface (D), insert shows hollowness of broken pillars. 


\section{CONCLUSION}

This paper introduced two approaches for developing a parylene $\mathrm{C}$ micropillar array for various surface chemistry or biology applications. The etching technique produced high aspect ratio micropillars $(H R \approx 6)$ with slightly conical pillars due to the limitation in the capabilities of the equipment we used. The ion-beam etching is dependent on the beam energy and space charge, which is locally changing due to high polymer thickness. The non-transparency of the silicon substrate also limits this platform's use for transmission microscopy. Additionally, the residues from the hard mask are a disadvantage that is hard to overcome without losing original shape. On the other hand, the development of parylene $\mathrm{C}$ micropillars by molding technique can create a transparent micropillar membrane. The proper optimization of the Bosch process can develop high aspect ratio parylene pillars $(H R \approx 10)$ with minimal scalloping effect. However, micropillars become more fragile and collapse. To conclude, the proper optimization of both techniques must be performed to know the limitations of methods used in this work to develop cylindrical HAR micropillars.

\section{ACKNOWLEDGEMENTS}

\section{The authors wish to acknowledge the financial support of the GACR Project Number GA19-04270Y from the Czech Republic and the internal grant of BUT (FKT-S-20-6215).}

\section{REFERENCES}

[1] ALAPAN, Y., ICOZ, K., GURKAN, U. A. Micro- and nanodevices integrated with biomolecular probes. Biotechnology Advances. 2015, vol. 33, no. 8, pp.1727-1743.

[2] SCHOEN, I., HU, W., KLOTZSCH, E., VOGEL, V. Probing Cellular Traction Forces by Micropillar Arrays: Contribution of Substrate Warping to Pillar Deflection. Nano Letters. 2010, vol. 10, no. 5, pp. 1823-1830.

[3] SHINOHARA, H., GOTO, H., KASAHARA, T., MIYUNO, J. Fabrication of a Polymer High-Aspect-Ratio Pillar Array Using UV Imprinting. Micromachines. 2013, vol.4, no. 2, pp. 157-167.

[4] PAPADOPOULOS, P., PINCHASIK, B.-E., TRESS, M., DORIS, V., KAPPL, M., BUTT, H.-J. Wetting of soft superhydrophobic micropillar arrays. Soft Matter. 2018, vol. 14, pp. 7429-7434.

[5] DE LA OLIVA, N., MUELLER, M., STIEGLITZ, T., NAVARRO, X., DEL VALLE, J. On the use of Parylene C polymer as substrate for peripheral nerve electrodes. Scientific Reports. 2018, vol. 8, no. 1, pp. 5965-5977.

[6] FOHLEROVA, Z., GABLECH, I., OTAHAL, A., FECKO, P. SiO2-Decorated Parylene C Micropillars Designed to Probe Cellular Force. Advanced Materials Interfaces. 2021, vol. 8, no. 6, pp. 2001897-2001907.

[7] LIU, X., FECKO, P., FOHLEROVA, Z., KARASEK, T., PEKAREK, J., NEUZIL, P. Parylene micropillars coated with thermally grown SiO2. Journal of Vacuum Science \& Technology B. 2020, vol. 38, no. 6, pp. 063001.

[8] ZHANG, L., LIU, Y., LI, Z., WANG, W. SF6 Optimized O2 Plasma Etching of Parylene C. Micromachines. 2018, vol. 9, no. 4, pp. 162-170.

[9] PARK, J. S., KANG, D.-H., KWAK, S. M., KIM, T. S., PARK, J. H., KIM, T. G., BAEK, S.-H., LEE, B. C. Lowtemperature smoothing method of scalloped DRIE trench by post-dry etching process based on SF6 plasma. Micro and Nano Systems Letters. 2020, vol. 8, no. 1, pp. 14-22. 